DOI: 10.12957/demetra.2017.26467

\title{
Disponibilidade espacial de peixarias em áreas de diferentes níveis socioeconômicos de uma cidade litorânea
}

\author{
Spatial availability of fish stores in areas of different socioeconomic levels of a coast city
}

\author{
Elizabeth Nappi Corrêa ${ }^{1}$ \\ Ângelo Horta de $\mathrm{Abreu}^{2}$ \\ Camila Elizandra Rossi \\ Cristine Garcia Gabriel' \\ Janaina das Neves ${ }^{3}$ \\ Maria Gabriela Matias de Pinho ${ }^{4}$ \\ Francisco de Assis Guedes de \\ Vasconcelos' \\ 1 Universidade Federal de Santa Catarina, \\ Departamento de Nutrição, Programa de Pós- \\ graduação em Nutrição. Florianópolis-SC, Brasil. \\ ${ }^{2}$ Faculdade de Letras da Universidade de \\ Coimbra, Departamento de Geografia, Programa \\ de Pós-graduação em Geografia Física e \\ Ordenamento do Território. Coimbra, Portugal. \\ ${ }^{3}$ Universidade Federal de Santa Catarina, \\ Departamento de Nutrição. Florianópolis-SC, \\ Brasil. \\ ${ }^{4}$ VU University Medical Center Amsterdam, \\ Departamento de Epidemiologia e Bioestatística. \\ Amsterdam, Holanda. \\ Correspondência / Correspondence \\ Elizabeth Nappi Corrêa \\ E-mail: nutrinappi@gmail.com
}

\section{Resumo}

Introdução: $\mathrm{O}$ consumo de alimentos in natura tem sido estimulado por diversas políticas públicas. Investigações sobre disponibilidade geográfica de locais que comercializam esses alimentos e sua relação com a qualidade da alimentação da população têm sido enfatizadas desde o início do século XXI. Objetivo: Identificar a distribuição espacial das peixarias fixas e itinerantes no município de Florianópolis, de acordo com a densidade de estabelecimentos por habitantes, em áreas geográficas de diferentes níveis de renda domiciliar. Metodologia: As informações foram obtidas por meio de dados secundários provenientes do setor de Vigilância Sanitária Municipal e de listas telefônicas digitais e impressas. Para a localização geográfica dos estabelecimentos nas 30 áreas de ponderação do município, foi utilizado o software Google Earth ${ }^{\circledast}$. Resultados e discussão: A distribuição espacial das peixarias fixas não contempla 14 (47\%) das áreas geográficas de Florianópolis, sendo 9 (64\%) atendidas pelo "Projeto Caminhão do Peixe", caracterizado pela comercialização itinerante de pescados. Apesar de não haver diferença estatística, observou-se que os números absoluto e relativo (por habitantes) das peixarias fixas eram mais altos quanto maior era a renda da área geográfica. Conclusões: Recomenda-se que futuros estudos procurem relacionar a distribuição ambiental das peixarias à aquisição e ao consumo de pescados em Florianópolis.

Palavras-chave: Comercialização de Produtos. Consumo de Alimentos. Peixes. Ambiente Alimentar. Mapeamento Geográfico. 


\section{Abstract}

Introduction: Fresh food intake has been stimulated through several public policies. Over the last decades, research has been conducted on the spatial availability of stores that sell this type of food and their relationship with the population's food consumption quality. Objective: This study identified the distribution of fixed and itinerant fish commerce in Florianópolis, a city located in Southern Brazil. Also, we seek to associate the density (ratio of number of fish stores by inhabitants) in areas of different income levels. Methodology: Data were collected through secondary data in the records of the Municipal Sanitary Surveillance and in digital and printed telephone directories. For data spatialization, we used the Google Earth software ${ }^{\circledR}$, which has shown the location of fish stores in 30 geographical areas. Results and discussion: The spatial distribution of fish marketing stores in Florianópolis does not address all the geographic areas, but the "Fish Truck", characterized by itinerant marketing of fish, serves most of them. Although there was no statistical difference, the absolute and relative numbers (per inhabitant) of the fixed fish trade were higher, the higher the income of the geographical area. Conclusion: Future studies should be conducted to relate the environmental distribution of fish stores to the purchase and consumption of fish in Florianópolis.

Keywords: Products Commerce. Food Consumption. Fishes. Food Environment. Geographic Mapping.

\section{Introdução}

Guias alimentares de distintos países têm recomendado o consumo regular de peixes e outros frutos do mar. A FAO recomenda a ingestão de $12 \mathrm{~kg}$ pescados per capita por ano. ${ }^{1}$ Esses alimentos são ricos em ácidos graxos essenciais insaturados e proteínas de alto valor biológico e, por consequência, são considerados saudáveis e cardioprotetores. ${ }^{2-6}$

Entre os países com maiores médias de consumo de proteínas provenientes de peixes (gramas/ pessoa/dia), pode-se citar: Japão (17,9 g/dia), República da Coreia (16,7 g/dia), Portugal (15,3 g/dia) e Noruega (15,1 g/dia). Os dados para o Brasil indicam consumo médio de 10,6 g/dia. ${ }^{7}$

O mercado brasileiro de pescados apresenta uma série de especificidades inter e intrarregionais decorrentes da diversidade sociocultural, da multiplicidade étnica e de aspectos econômicos que influenciam os hábitos alimentares. ${ }^{8}$ Por isso, a aquisição per capita por ano de peixes e demais 
pescados no Brasil tem sofrido flutuações, estando sempre abaixo da recomendação de consumo mundial. As últimas Pesquisas de Orçamento Familiar (POF) realizadas no país nos anos de 20022003 e 2008-2009 identificaram redução na aquisição anual per capita de pescados, passando de 4,59 $\mathrm{kg}^{9}$ para 4,03 kg, ${ }^{10}$ respectivamente, correspondendo ao consumo aparente desse tipo de alimento.

A região Sul do Brasil, mesmo contando com dois portos pesqueiros (Itajaí e São Francisco do Sul), observou diminuição de $50 \%$ na aquisição média anual per capita de pescados nos últimos 30 anos. ${ }^{11}$ Na década de 1970, correspondia a 3,2 kg/ano. ${ }^{12}$ No final da primeira década do século XXI, esse valor caiu para $1,59 \mathrm{~kg} / \mathrm{ano}$, a menor aquisição per capita de pescados do país. Especificamente em Santa Catarina, estado cuja capital Florianópolis situa-se quase que completamente em uma ilha, a aquisição per capita de pescados no ano de 2008-2009 correspondeu a apenas 1,96 kg/ano. ${ }^{11}$

Esta aquisição de baixas proporções, mesmo em território provido de região litorânea expressiva, aponta para a necessidade de se avaliar a disponibilidade espacial de locais de comercialização de pescados, como as peixarias, visto que o acesso físico aos locais de comercialização de alimentos pode influenciar distintos públicos, incluindo adultos, crianças e adolescentes, a adquirirem e/ ou consumirem produtos por eles vendidos, sejam saudáveis ou não. ${ }^{13-15}$ Por exemplo, uma maior acessibilidade ambiental a restaurantes do tipo fast-food vem sendo associada com o seu consumo e com dietas de baixo valor nutricional. ${ }^{16-18}$ Por outro lado, pessoas vivendo em ambientes com maior acesso a locais que vendem alimentos saudáveis são mais propensas a ter uma dieta de melhor qualidade nutricional..$^{19}$ Em relação aos adultos, esses residindo próximos a pequenos mercados e mercearias, tendem a consumir mais frutas, legumes e verduras. ${ }^{20}$ Porém, quando esses estabelecimentos são supermercados, os resultados ainda são divergentes, mostrando-se diferentes conforme níveis socioeconômicos das áreas avaliadas e países estudados. ${ }^{21-23}$ De modo geral, os estudos sobre a disponibilidade não têm abordado os pescados como objeto de análise.

Dessa forma, aliado à avaliação da acessibilidade física, vale investigar as distribuições espaciais dos estabelecimentos por níveis socioeconômicos, uma vez que tanto a oferta quanto a compra de alimentos podem estar restritas em áreas cujos habitantes tenham insuficiência de recursos financeiros, afetando negativamente a qualidade da dieta dos seus residentes. ${ }^{24}$

Os pescados podem ser adquiridos em supermercados, peixarias e feiras livres, porém, desde a década de 1980, os supermercados vêm se tornando o principal canal de comercialização de alimentos para o consumidor final. ${ }^{25}$ Entretanto, existem diferenças entre o perfil dos consumidores. Sonoda, ${ }^{25}$ ao avaliar a demanda por pescados no Brasil entre 2002-2003, observou que os supermercados e as peixarias atendiam a públicos de diferentes níveis de renda. Enquanto os mais favorecidos economicamente procuram os supermercados para adquirirem os pescados, as peixarias são a preferência das classes sociais menos favorecidas. 
Considerando-se a importância do consumo de pescados para a saúde humana e a escassez de estudos que mostrem a disponibilidade espacial de peixarias e sua relação com dieta e compra de alimentos, o objetivo deste artigo foi identificar a distribuição espacial das peixarias no município de Florianópolis, de acordo com a densidade de estabelecimentos por habitantes em áreas geográficas de diferentes níveis de renda domiciliar.

\section{Métodos}

Trata-se de estudo do tipo transversal descritivo, realizado a partir da utilização de dados secundários relativos à quantidade e distribuição de peixarias fixas e itinerantes no município de Florianópolis.

\section{Caracterização do local do estudo}

Florianópolis é um município situado no sul do Brasil, formado, em sua maior parte, por uma ilha (97\%). Destaca-se no cenário nacional pelo elevado Índice de Desenvolvimento Humano Municipal (IDHM), correspondendo a 0,847 (muito alto), em 2010, superior ao IDH nacional no mesmo ano considerado alto $(0,727) \cdot{ }^{26}$

A população estimada para o município de Florianópolis no ano de 2016 é de 477.798 habitantes, distribuídos em um território de $675.409 \mathrm{~km}^{2}$, resultando em densidade populacional de 683,3 habitantes $/ \mathrm{km}^{2}$, com $96,2 \%$ da população em área urbana. De acordo com o Censo 2010, Florianópolis apresenta 651 setores na malha censitária (605 na área urbana e 46 na área rural), agregados em 30 áreas de ponderação. ${ }^{27}$ Áreas de ponderação são agrupamentos de setores censitários, identificadas pelo Instituto Brasileiro de Geografia e Estatística (IBGE) como unidades mínimas de análises, ao se estudar populações.

\section{Coleta e análise dos dados}

Neste estudo, foi realizado o mapeamento de locais de comercialização de pescados no município de Florianópolis, sendo consideradas as peixarias fixas e o "Projeto Caminhão do Peixe" (peixaria itinerante), o qual consiste na distribuição de caminhões-feira, pelo Ministério da Pesca e da Aquicultura (MPA). Desde 2009, vende pescados diretamente aos consumidores, a preços mais acessíveis que os pontos fixos de comercialização. ${ }^{28}$

Os dados de localização das peixarias foram capturados de fontes secundárias, que, em comparação a fontes de dados geográficos primários, podem ter, relativamente, baixo custo de obtenção e geralmente abranger uma grande área geográfica (por exemplo, o âmbito municipal). ${ }^{29}$ 
Para a identificação das peixarias fixas (estabelecidas em endereços formais), foi solicitado o cadastro da Gerência de Vigilância Sanitária e Ambiental da Secretaria da Saúde da Prefeitura Municipal de Florianópolis, contendo as informações sobre os estabelecimentos do município que comercializavam alimentos no ano de 2013. Dessa listagem foram identificados o nome e o endereço (rua, bairro e CEP) das peixarias. Entretanto, para garantir confiabilidade, também foi feita a triangulação das informações por meio de diferentes fontes de dados, visando à complementação e conferência desses endereços. ${ }^{30}$ Para tal, foi realizada consulta à lista telefônica impressa distribuída no município e ao cadastro dos estabelecimentos comerciais de alimentos em listas telefônicas disponibilizadas no formato on-line, ${ }^{\text {a }}$ sendo utilizado o verbete "peixarias" na ferramenta de busca.

Para a identificação das peixarias itinerantes de Florianópolis, consultou-se o site da Secretaria Municipal de Pesca, Maricultura e Agricultura de Florianópolis, ${ }^{b}$ órgão responsável pelas ações relacionadas à atividade pesqueira no município. Nessa página eletrônica da Secretaria é disponibilizada bimestralmente a agenda do "Projeto Caminhão do Peixe", iniciativa que visa aumentar a comercialização e incentivar o consumo de pescados. Na agenda, é possível identificar o local de instalação das peixarias itinerantes que atendem aos bairros de Florianópolis.

Após essas etapas da identificação dos endereços das peixarias fixas e itinerantes, foi realizada a conferência do CEP e da grafia correta e completa dos endereços por meio do site oficial da Empresa Brasileira de Correios e Telégrafos. ${ }^{c}$ De posse dessas informações, efetuou-se o processo de espacialização manual das peixarias do município, utilizando-se o software Google Earth ${ }^{\circledR}$, ou seja, os endereços completos desses estabelecimentos foram manualmente espacializados (criação de referência espacial com base nas coordenadas geográficas). Durante esse processo, o recurso Street View ${ }^{\circledR}$ foi usado para identificar com maior precisão a localização dos estabelecimentos.

Para caracterizar o perfil de distribuição das peixarias do município, foram obtidos dados demográficos e socioeconômicos dos residentes de Florianópolis a partir do Censo 2010 e agregados em áreas de ponderação. ${ }^{27}$ De acordo com o IBGE, os dados demográficos dos setores censitários não podem ser usados por causa da não representatividade estatística. Nesse caso, a unidade mínima de análise proposta pelo próprio IBGE é a área de ponderação, constituída por um agrupamento de setores censitários, nos quais os resultados apresentam significância estatística e podem ser usados para análises acadêmicas. ${ }^{27}$

\footnotetext{
a http://www.hagah.com.br/; http://www.guiafacil.com/florianopolis/sc/; http://www.telelistas.net/sc/ florianopolis

b http://www.pmf.sc.gov.br/entidades/pesca

c http://www.buscacep.correios.com.br/
} 
Os resultados são apresentados na forma de estatística descritiva, a partir da distribuição das peixarias fixas e itinerantes por três classes de renda domiciliar. No agrupamento das informações e análises das áreas de ponderação, optou-se por trabalhar com a distribuição do tercil de renda média nominal mensal dos domicílios, obtendo-se: $1^{\circ}$ tercil de distribuição (U\$ 1.395 - U\$2.180), $2^{0}$ tercil de renda (U\$2.181 - U\$3.021) e $3^{0}$ tercil de renda (U\$3.022 - U\$ 6.165). A média do rendimento nominal mensal encontrada foi de $\mathrm{U} \$ 2.903$. Os valores do rendimento nominal médio são apresentados pelo IBGE em reais (R\$), entretanto, neste artigo estes valores são descritos em dólar (U\$). Para a conversão foi considerada a cotação média do dólar em agosto de 2010 no valor de, aproximadamente, $\mathrm{R} \$ 1,75$, período inicial da coleta de dados do Censo de 2010. ${ }^{27}$

A densidade de peixarias, fixas e itinerantes, foi calculada por mil habitantes em cada área de ponderação, sendo também apresentada a comparação dessas densidades nas áreas residenciais por meio do teste não paramétrico de tendência, considerando 5\% de nível de significância estatística.

\section{Resultados}

Na tabela 1 estão identificadas as 30 áreas de ponderação do município por tercil de renda e a quantidade de peixarias fixas e itinerantes em cada uma delas, bem como a densidade das mesmas por 1.000 habitantes. Das 30 áreas existentes, 46,7\% $(n=14)$ não possuem peixarias fixas, seis delas se localizando em áreas do tercil mais baixo de renda e outras seis em áreas de mais alto tercil de renda (dados não apresentados).

Tabela 1. Distribuição das peixarias por áreas de ponderação, conforme tercis de renda domiciliar nominal mensal e densidade das peixarias por mil habitantes - Florianópolis, 2013.

\begin{tabular}{ccccc}
\hline $\begin{array}{c}\text { Áreas de ponderação } \\
\text { segundo tercis de renda }\end{array}$ & $\begin{array}{c}\text { Peixarias } \\
\text { fixas }\end{array}$ & $\begin{array}{c}\text { Densidade por } \\
1.000 / \text { hab. }\end{array}$ & $\begin{array}{c}\text { Peixaria } \\
\text { itinerante }\end{array}$ & $\begin{array}{c}\text { Densidade por } \\
1.000 / \text { hab. }\end{array}$ \\
\hline $\begin{array}{c}\text { Áreas de ponderação de } \\
\text { menor renda }\end{array}$ & 6 & $0,01^{*}$ & 7 & $0,05^{* *}$ \\
$\begin{array}{c}\text { Áreas de ponderação de } \\
\text { renda intermediária }\end{array}$ & 12 & $0,08^{*}$ & 13 & $0,09 * *$ \\
$\begin{array}{c}\text { Áreas de ponderação de } \\
\text { maior renda }\end{array}$ & 16 & $0,11^{*}$ & 5 & $0,04^{* *}$ \\
\hline
\end{tabular}

Teste não paramétrico de tendência *p 0,$811 ; * *$ p 0,094 . 
Observa-se também que o número e a densidade de peixarias fixas aumentam proporcionalmente à renda domiciliar da área, porém sem significância estatística. A densidade de peixarias fixas no tercil mais alto de renda se destaca devido à presença do mercado público municipal na área central do município, que comporta 13 das 34 peixarias do município (38\%).

A distribuição geográfica das 34 peixarias fixas nas áreas de ponderação por tercil de renda pode ser visualizada na figura 1.

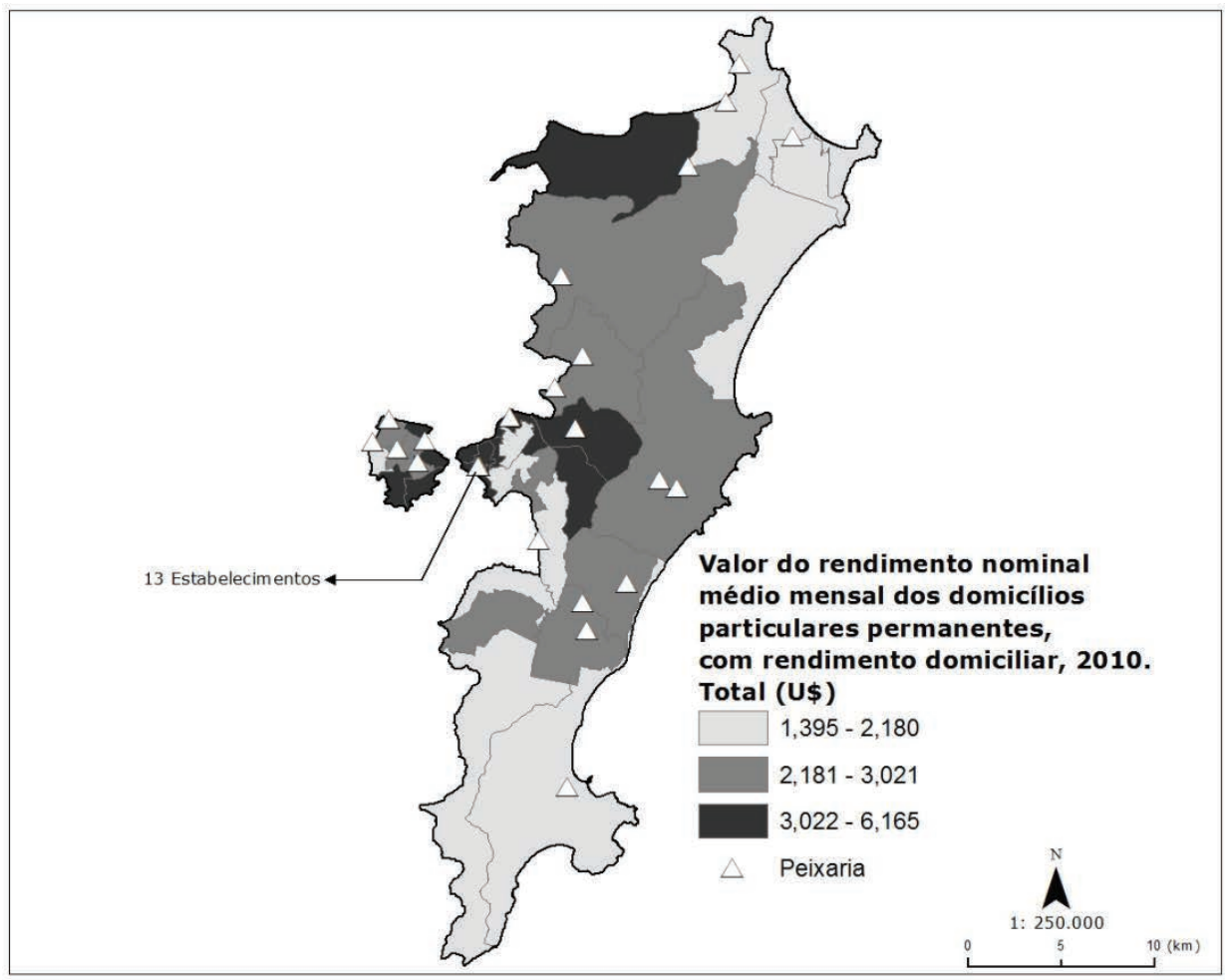

Áreas de Ponderação de Florianópolis

Figura 1. Áreas de ponderação de Florianópolis de acordo com o rendimento nominal médio mensal dos domićlios particulares em dólar no ano de 2010 e a localização das peixarias. Florianópolis, Santa Catarina, Brasil, 2013. 


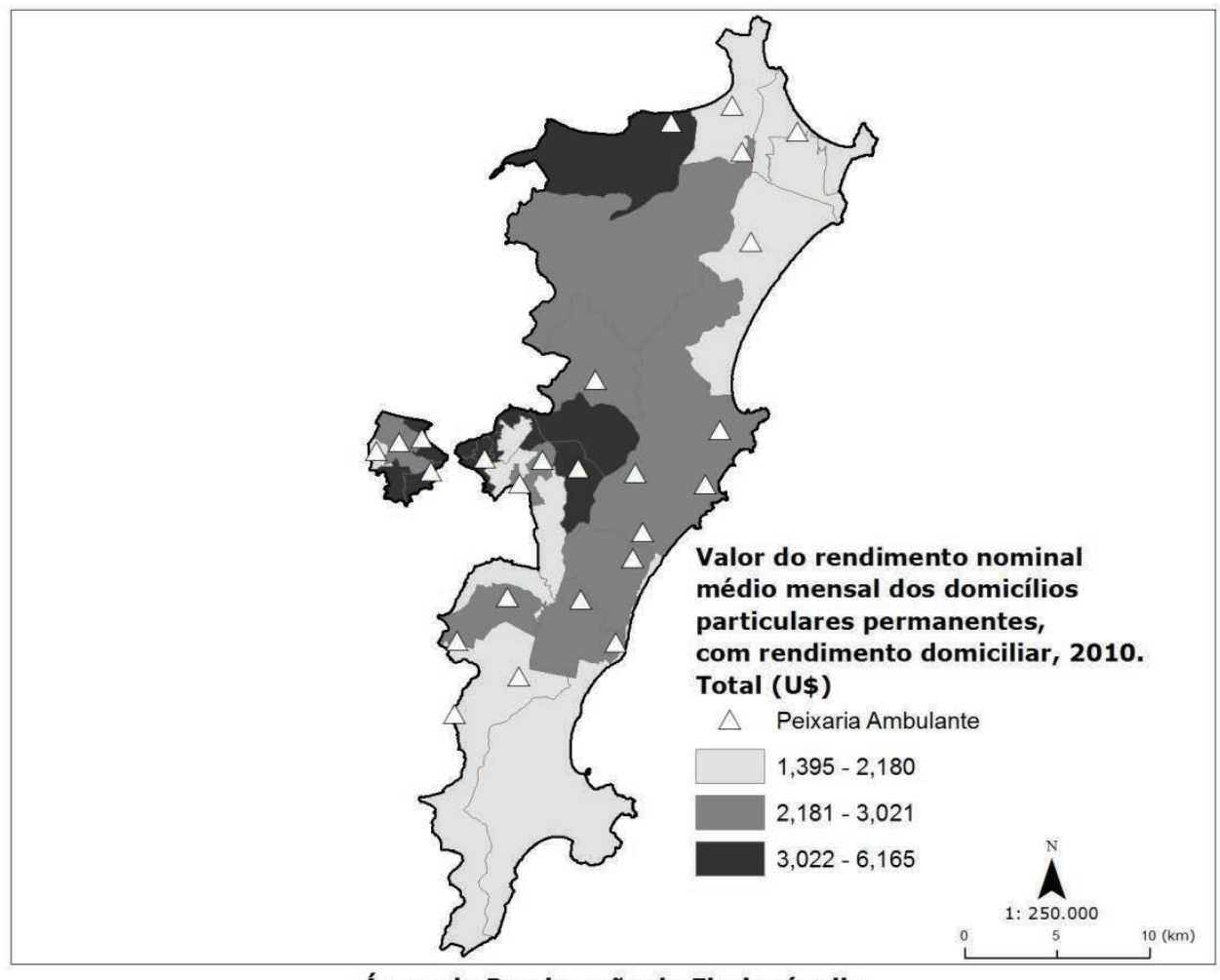

Áreas de Ponderação de Florianópolis

Figura 2. Áreas de ponderação de Florianópolis de acordo com o rendimento nominal médio mensal dos domicílios particulares em dólar no ano de 2010 e a localização das peixarias itinerantes. Florianópolis, Santa Catarina, Brasil, 2013.

Na figura 2 é possível identificar a distribuição geográfica de 25 pontos de comercialização da peixaria itinerante de Florianópolis, caracterizando a rota do "Caminhão do Peixe". A densidade desses estabelecimentos por habitantes é menor que aquela das peixarias fixas e, ao contrário do observado nas peixarias fixas, a densidade das itinerantes por população não é diretamente proporcional à renda domiciliar das áreas. A maior das densidades se encontra em áreas do tercil médio de renda, ainda que não haja diferenças significativas. Segundo o portal da Prefeitura Municipal de Florianópolis, nesses estabelecimentos são comercializados peixes congelados, inteiros ou em postas, filés, bandejas de bolinhos de siri, de peixe e de camarão, totalizando 19 tipos de produtos em bandejas de $1,0 \mathrm{~kg}$.

Ao comparar a distribuição espacial das peixarias fixas e das itinerantes, é possível perceber que entre as 14 áreas residenciais onde inexistem peixarias fixas, nove (64,3\%) são cobertas pela 
peixaria itinerante. Entre essas nove, quatro estão localizadas em regiões de mais alto tercil de renda, enquanto três delas estão nas regiões de mais baixo tercil de renda.

\section{Discussão}

Neste artigo, identificou-se a distribuição espacial de peixarias fixas e itinerantes no município de Florianópolis de acordo com a densidade de estabelecimentos por habitantes, em locais de diferentes níveis de renda domiciliar.

Os resultados mostraram inexistência de peixarias fixas em quase metade das áreas de ponderação, o que pode caracterizar deserto alimentar e consequente redução de oportunidades para o consumo de pescados. O termo deserto alimentar tem sido utilizado para descrever a ausência de pontos de venda de alimentos em uma área definida ${ }^{31}$ e pode indicar uma barreira para a aquisição de determinados itens alimentares. ${ }^{32}$ Ressalta-se, contudo, que, apesar de este estudo ter focado na avaliação da disponibilidade de peixarias, que é o espaço preferido por 13\% da população brasileira, ${ }^{32}$ e por igual proporção dos consumidores de pescado em Rio Grande/RS, ${ }^{33}$ outros espaços para compra de pescados também podem estar sendo utilizados pela população de Florianópolis. Na amostra nacional, por exemplo, 24,5\% dos brasileiros relataram adquirir pescados no supermercado/hipermercado ${ }^{32}$ e, em Rio Grande, $64 \%$ dos respondentes citaram comprá-los em mercados, sendo 39\% deles convencionais e 25\%. públicos. ${ }^{33}$ Então, devido à existência desses outros pontos de aquisição de pescado, à presença das peixarias itinerantes em Florianópolis, que suprem nove $(64,3 \%$ ) áreas de deserto de peixarias fixas, e ao fato de um terço dos pontos de venda itinerantes se localizar em regiões do mais baixo tercil de renda, acredita-se que a oportunidade para aquisição de pescados em Florianópolis possa se concretizar em outros espaços do ambiente alimentar, como os mercados, supermercados e peixarias itinerantes.

Entretanto, os achados também mostraram uma tendência de aumento na densidade de peixarias fixas conforme aumento dos tercis de renda nas áreas geográficas. Este resultado pode ser decorrente da demanda de aquisição de pescados, que, segundo a POF 2008-2009, mostra-se diferente nas distintas classes de renda. A compra de pescados em geral entre as famílias com mais baixa renda mensal foi em torno de $0,606 \mathrm{~kg}$ per capita por ano. Já entre as famílias com mais alta renda mensal, a compra ultrapassou os $3,5 \mathrm{~kg}$. ${ }^{11}$ Porém, há que se considerar também que os públicos de menor renda tendem a ser bastante exigentes quanto aos preços e optam pelo não congelamento dos pescados, ${ }^{8,34}$ acessando preferencialmente as peixarias, ${ }^{25}$ possivelmente para sanar estes dois quesitos de compra. Considerando, ainda, que predomina no mercado brasileiro a preferência do consumidor pelo pescado sazonal, condizente com a cultura alimentar, ${ }^{8}$ reforça-se a importância das peixarias, que parecem oportunizar a compra de pescados aos públicos de renda menos elevada. 
Em Florianópolis, moradores de 25 bairros são atendidos pelas peixarias itinerantes, entretanto, as localidades selecionadas podem variar a cada bimestre, de acordo com solicitação das comunidades e com a procura pelos residentes em cada localidade. O "Caminhão do Peixe" percorre os bairros durante o turno matutino e em dias úteis, sendo assim, a periodicidade com que a peixaria itinerante percorre cada um dos bairros é de cerca de 40 dias, o que pode limitar a aquisição e, consequentemente, o consumo por parte da população. É importante ressaltar que o projeto propõe a comercialização de produtos provenientes da pesca artesanal e da aquicultura familiar, minimizando a ação de intermediários. ${ }^{28}$

Vale destacar também o Mercado Público de Florianópolis, que, além de ser patrimônio arquitetônico, é ponto tradicional de comercialização de pescados. Na área de ponderação em que se localiza o mercado, encontra-se a maior densidade de peixarias por habitantes (1,18 para 1.000/habitantes), o que possivelmente contribui para a aquisição de pescados pela população. Contudo, pela concentração de peixarias na mesma edificação, pode-se também sugerir que o acesso à compra é geograficamente restrito, pois as pessoas que querem adquirir peixes na área central de Florianópolis têm apenas um ponto de referência. Ao contrário, considerando-se que em Florianópolis algumas das áreas de ponderação podem abrigar cooperativas de pescadores e que talvez por esse motivo não disponham de peixarias, cita-se a implementação do Plano Safra da Aquicultura e Pesca 2012/2013/2014, que previu investimentos para expandir a aquicultura, modernizar a pesca e fortalecer a indústria e o comércio pesqueiro. ${ }^{35}$ Ainda, os Planos Nacionais de Segurança Alimentar e Nutricional (PLANSAN 2012/2015 e 2016/2019) destacam a necessidade de adequada assistência técnica e de inovação tecnológica para os pescadores artesanais e aquicultores familiares, visando à inclusão produtiva desses e a ampliação e qualificação do abastecimento de pescado para o consumo interno e os mercados institucionais, com foco no Programa de Aquisição de Alimentos (PAA) e no PNAE. Entre as ações prioritárias, sobressai a expansão de pontos comerciais, como as peixarias. ${ }^{36} \mathrm{O}$ estado de Santa Catarina publicou seu 1ํ Plano Estadual de Segurança Alimentar e Nutricional (2014/2019), reforçando as diretrizes nacionais e destacando a necessidade da criação e regulamentação de legislação específica para agricultura e pesca familiares. ${ }^{37}$ Uma vez que os planos citados são instrumentos de gestão recentes e demandam ações inovadoras, complexas, integradas e intersetoriais, tornam-se necessários mecanismos de contínua avaliação, monitoramento e ação-reflexão-ação sobre as problemáticas e caminhos propostos. Espera-se, com essas iniciativas públicas, que os pontos de venda de pescados in loco também tenham sido incentivados.

Por fim, os resultados encontrados e discutidos neste estudo apontam para uma avaliação mais abrangente dos pontos de venda de pescados em Florianópolis, para se confirmar a necessidade de contemplar de forma mais homogênea a venda de pescados no município, atingindo, principalmente, as áreas de renda menos favorecidas. Isso deve ser possibilitado mediante políticas 
públicas eficazes, supracitadas, que levem em consideração as características populacionais, culturais e hábitos alimentares dos florianopolitanos, implantadas após realização de mais trabalhos semelhantes a este. Os resultados encontrados podem ser utilizados para comparação de estudos realizados em outras regiões do país.

Assim, ainda que a presente investigação não tenha avaliado os supermercados, mercearias e a compra direta do pescador, ele foi capaz de identificar as peixarias, o Mercado Público Municipal (localizado na área central do município) e a peixaria itinerante ("Projeto Caminhão do Peixe"). Considerando-se o vasto litoral brasileiro, acredita-se que estudos com esta característica possam ser replicados e difundidos para outras localidades, fornecendo subsídios para o melhor planejamento e utilização dos espaços que conformam o ambiente alimentar.

Sugere-se, ainda, que estudos subsequentes em Florianópolis averiguem quais são os espaços de preferência para compra de pescados no município, em famílias de diferentes níveis de renda, e quais as quantidades consumidas. Ainda, é importante compilar os tipos de pescados adquiridos por essa população e identificar se a frequência bimestral da peixaria itinerante tem impacto na aquisição e no consumo de pescados, bem como se faz necessário investigar os motivos pelos quais atualmente são comercializados pescados congelados e manufaturados provenientes de fora do município no "Caminhão do Peixe" (dados não mostrados, porém, relatados pela Secretaria da Pesca do município), ao contrário do que recomendam as ações desta iniciativa pública.

\section{Conclusão}

A distribuição espacial das 34 peixarias fixas em Florianópolis não atende às 30 áreas de ponderação, sugerindo baixo acesso às mesmas e necessidade de se estudar o consumo de pescados na população do município. Algumas dessas áreas foram atendidas pelo "Caminhão do Peixe", caracterizado pela comercialização itinerante de pescados, o qual, no entanto, está disponível com periodicidade bimestral em cada um dos 25 bairros que percorre.

A densidade de peixarias fixas por habitantes aumenta conforme a renda média mensal das áreas de ponderação, fato que suscita investigações sobre tipos de mercados de peixe acessados por famílias menos e mais favorecidas economicamente.

Por fim, sugere-se que sejam implementadas ações que possam contribuir efetivamente para o acesso ao consumo de peixe/pescado. Talvez uma delas seja a ampliação das rotas e da frequência do “Caminhão do Peixe”. Recomenda-se ainda que futuros estudos procurem relacionar a distribuição ambiental das peixarias à aquisição e ao consumo de pescados em Florianópolis. 


\section{Colaboradores}

Corrêa EN trabalhou em todas as etapas desde a concepção do estudo, coleta de dados, análise e interpretação dos dados até a revisão da versão final do artigo; de Abreu AH colaborou com a análise e interpretação dos dados; Rossi CE participou da análise dos dados, redação do artigo e da sua versão final; Gabriel CG participou da análise dos dados, da redação do artigo e da sua versão final; Neves J participou da redação do artigo e da sua versão final; de Pinho MGM trabalhou na concepção do estudo, na análise dos dados e na redação do artigo; de Vasconcelos FAG participou do desenho do estudo e da revisão da versão final do artigo.

Todos os autores deste artigo são integrantes de projeto de pesquisa financiado e participaram ativamente de várias etapas do estudo. Além disso, destacamos a participação de dois pesquisadores do grupo de trabalho com vínculo com outras universidades (Universidade de Coimbra e VU University), fortalecendo no grupo parcerias institucionais importantes.

Conflito de Interesses: Os autores declaram não haver conflito de interesses.

\section{Referências}

1. Food and Agriculture Organization of the United Nations. La producción mundial de pescado, crustáceos y moluscos llegó a 126,2 millones de toneladas em 1999, es decir un incremento del 7 por ciento em comparación con el nivel de 1998. [acesso em: 27 abr. 2004]. Disponível em: http://www. fao.org/docrep/005/Y7300S/y7300s05.htm

2. US. Department of Agriculture; U.S. Department of Health and Human Services. Dietary guidelines for americans 2010. Washington, DC: Government Printing Office; 2010.

3. Krauss RM, Eckel RH, Howard B, Appel LJ, Daniels SR, Deckekbaum RJ, et al. AHA Dietary Guidelines. Revision 2000: A statement for healthcare professionals from the Nutrition Committee of the American Heart Association. Circulation 2000; 102: 2284-2299.

4. Food and Agriculture Organization of the United Nations. The state of world fisheries and aquaculture 2012. Rome: WHO, FAO; 2012.

5. Brasil. Ministério da Saúde. Guia alimentar para a população brasileira: promovendo a alimentação saudável. Brasília: Ministério da Saúde; 2006.

6. Brasil. Ministério da Saúde. Guia alimentar para a população brasileira. Brasília: Ministério da Saúde; 2014.

7. Food and Agriculture Organization of the United Nations. World Health Organization. Report of the Joint FAO/WHO Expert Consultation on the Risks and Benefits of Fish Consumption. Geneva: FAO, WHO; 2010.

8. Soares ALS. Aspectos econômicos do mercado brasileiro de pescado. [acesso em: 12 set. 2016]. Disponível em: ftp://ftp.sp.gov.br/ftppesca/3simcope/3simcope_palestra3.pdf

9. Instituto Brasileiro de Geografia e Estatística. Pesquisa de Orçamentos Familiares 1986 - 1987.Rio de Janeiro: IBGE; 1987. 
10. Instituto Brasileiro de Geografia e Estatística. Pesquisa de Orçamentos Familiares (POF) 1995 - 1996. Rio de Janeiro:IBGE; 1996.

11. Instituto Brasileiro de Geografia e Estatística. Pesquisa de Orçamentos Familiares 2008 - 2009. Rio de Janeiro: IBGE; 2010.

12. InstitutoBrasileiro de Geografia e Estatística. Estudo Nacional de Despesa Familiar. Rio de Janeiro: IBGE; 1978.

13. Giskes K, Van Lenthe F, Avendano-Pabon M, Brug J. A systematic review of environmental factors and obesogenic dietary intakes among adults: are we getting closer to understanding obesogenic environments? Obes Rev. 2011; 12(5):e95-e106.

14. Carroll-Scott A, Gilstad-Hayden K, Rosenthal L, Peters SM, McCaslin C, Joyce R, et al. Disentangling neighborhood contextual associations with child body mass index, diet, and physical activity: the role of built, socioeconomic, and social environments. Soc Sci Med. 2013; 95:106-14.

15. Corrêa EN, Schmitz BAS, Vasconcelos FAG. Aspects of the built environment associated with obesity in children and adolescents: a narrative review. Rev Nutr. 2015; 28(3):327-340.

16. Larson NI, Story MT, Nelson MC. Neighborhood environments: disparities in access to healthy foods in the U.S. Am J Prev Med. 2009; 36(1):74-81.

17. Burgoine T, Forouhi NG, Griffin SJ, Wareham NJ, Monsivais P. Associations between exposure to takeaway food outlets, takeaway food consumption, and body weight in Cambridgeshire, UK: population based, cross sectional study. BMJ 2014; 348:g1464.

18. Richardson AS, Meyer KA, Howard AG, Boone-Heinonen J, Popkin BM, Evenson KR, et al. Multiple pathways from the neighborhood food environment to increased body mass index through dietary behaviors: a structural equation-based analysis in the CARDIA study. Health Place 2015; 36:74-87.

19. Sallis JF, Glanz K. Physical activity and food environments: solutions to the obesity epidemic. Milbank Q 2009; 87(1):123-154.

20. Wedick NM, Yunsheng MA, Olendzki BC, Procter-Gray E, Cheng J, Kane KJ, et al. Access to healthy food stores modifies effect of a dietary intervention. Am J Prev Med. 2015; 48(3):309-17.

21. Skidmore P, Welch A, Van Sluijs E, Jones A, Harvey F, Harrison F, et al. Impact of neighbourhood food environment on food consumption in children aged 9-10 years in the UK SPEEDY (Sport, Physical Activity and Eating behaviour: Environmental Determinants in Young people) study. Public Health Nutr 2010;13(7):1022-30.

22. Boone-Heinonen J, Gordon-Larsen P, Kiefe CI, Shikany JM, Lewis CE, Popkin BM. Fast food restaurants and food stores: longitudinal associations with diet in young to middle-aged adults: the CARDIA study. Arch Intern Med. 2011; 171(13):1162-1170.

23. Vedovato GM, Trude AC, Kharmats AY, Martins PA. Degree of food processing of household acquisition patterns in a Brazilian urban area is related to food buying preferences and perceived food environment. Appetite 2015; 87:296-302.

24. Zenk SN, Schulz AJ, Israel BA, James SA, Bao S, Wilson ML. Neighborhood racial composition, neighborhood poverty, and the spatial accessibility of supermarkets in metropolitan Detroit. Am J Public Health 2005; 95(4):660-7. 
25. Sonoda DY. Demanda por pescados no Brasil entre 2002 e 2003 [tese]. [Piracicaba]: Escola Superior de Agricultura Luis de Queiroz; 2006.

26. Programa das Nações Unidas para o Desenvolvimento. Instituto de Pesquisa Econômica Aplicada. Fundação João Pinheiro. O Índice de Desenvolvimento Humano Municipal Brasileiro. Brasília: PNUD, IPEA, FJP; 2013.

27. Instituto Brasileiro de Geografia e Estatística. Censo demográfico 2010: características da população e dos domicílios. Rio de Janeiro: IBGE; 2011.

28. Brasil. Ministério da Pesca e Agricultura. Caminhões feira do peixe. Brasília: Ministério da Pesca e Agricultura; 2015.

29. Thornton LE, Pearce JR, Kavanagh AM. Using Geographic Information Systems (GIS) to assess the role of the built environment in influencing obesity: a glossary. Intern J Behav Nutr and Phys Act 2011; 8:71.

30. Wang MC, Kim S, Gonzalez AA, LacLeod KE, Winkleby MA. Socioeconomic and food-related physical characteristics of the neighbourhood environment are associated with body mass index. J Epidemiol Community Health 2007; 61(6):491-8.

31. Beaulac J, Kristjansson E, Cummins S. A systematic review of food deserts, 1966-2007. Prev Chronic Dis. 2009; 6(3):A105.

32. Instituto Brasileiro de Geografia e Estatística. Pesquisa de Orçamentos Familiares 2002 - 2003. Rio de Janeiro: IBGE; 2003.

33. Silveira LS, Abdallah PR, Hellebrandt L, Barbosa MN, Feijó FT. Análise socioeconômica do perfil dos consumidores de pescado no município de Rio Grande. Sinergia 2012;16(1):9-19.

34. Barros AR. Fundamentos econômicos da dinâmica da pesca em Pernambuco. Revista Econômica do Nordeste 2001; 32:569-591.

35. Brasil. Ministério da Pesca e Aquicultura. Plano Safra da Pesca e Aquicultura [Internet]. Brasília: MPA; 2014. [acesso em: 26 out. 2016]. Disponível em: http://docplayer.com.br/16688838-Planosafra-da-pesca-e-aquicultura-2012-2013-2014.html

36. Brasil. Ministério da Pesca e Aquicultura. MPA trabalha para inclusão do pescado na alimentação escolar [Internet] 27 abr. 2015 [acesso em: 10 nov. 2016]. Disponível em: http://www4.planalto.gov.br/consea/ comunicacao/noticias/2015/abril/mpa-trabalha-para-inclusao-do-pescado-na-alimentacao-escolar

37. Santa Catarina. Secretaria de Estado da Assistência Social. Trabalho e Habitação. Câmara Intersetorial de Segurança Alimentar do Estado de Santa Catarina. Plano Estadual de Segurança Alimentar e Nutricional: Santa Catarina 2014-2019. Florianópolis: Câmara Intersetorial de Segurança Alimentar do Estado de Santa Catarina; 2014.

Recebido: 24/11/2016

Revisado: $16 / 02 / 2017$

Aceito: 20/2/2017 